\title{
DESIGN AND PERFORMANCE ANALYSIS OF LOW POWER BAND STOP FILTER
}

\author{
Sarah Khwaja \\ Bachelor of Engineering from Jamia Millia Islamia, India
}

\begin{abstract}
In this paper, design and simulation of High performance Band-stop filter based on CNTFET-COTA using $45 \mathrm{~nm}$ technology node is proposed. The Cascade Operational Trans conductance amplifier (COTA) is an amplifier whose differential input voltage produces an output current. It is a voltage controlled current source (VCCS). Further, CNT technology is used to design and simulate proposed structure at $45 \mathrm{~nm}$ technology node. The proposed structure uses carbon nanotube field effect transistor. In a CNTFET, the channel is made up of parallel combination of SWCNTs. It is observed that the proposed BSF is also consuming low power of $42 \mu \mathrm{W}$.
\end{abstract}

Keywords: CMOS, CNTFET, DC Gain, Cascode -COTA, Power consumption, Filters, BPF.

Cite this Article: Sarah Khwaja, Design and Performance Analysis of Low Power Band Stop Filter, International Journal of Advanced Research in Engineering and Technology, 10 (6), 2019, pp 16-21.

http://iaeme.com/Home/issue/IJARET?Volume=10\&Issue $=6$

\section{INTRODUCTION}

Cascode OTA is a new class of OTA. It has improved gain (due to Band output resistance) and bandwidth due to reduced Miller capacitance [1-5]. In order to ensure further improvement in Cascode COTA performance, we have proposed Carbon Nanotube Field Effect Transistors (CNTFETs) based Circuits that promise to deliver much better performance than existing CMOS based Cascode Operational transconductance amplifiers [6]. CNTFET technology can easily club with the bulk CMOS technology on a single chip and utilizes the same infrastructure at $45 \mathrm{~nm}$ [7].

\section{DESIGN OF BAND STOP FILTER}

A filter with effectively the opposite function of the band pass is the band stop or notch filter. Notch filters are used to remove an unwanted frequency from a signal, while affect -ing all other frequencies as little as possible. It is formed by the combination of low pass and high pass filters with a parallel connection instead of cascading connection.

Since it eliminates frequencies, it is also called as band elimination filter or band reject filter or notch filter. 
Unlike high pass and low pass filters, band pass and band stop filters have two cut-off frequencies. It will pass above and below a particular range of frequencies whose cut off frequencies are predetermined depending upon the value of the components used in the circuit design. Any frequencies in between these two cut-off frequencies are attenuated.

When the input signal is given, the low frequencies are passed through the low pass filter in the band stop circuit and the high frequencies are passed through the high pass filter in the circuit. This is shown in below block diagram.

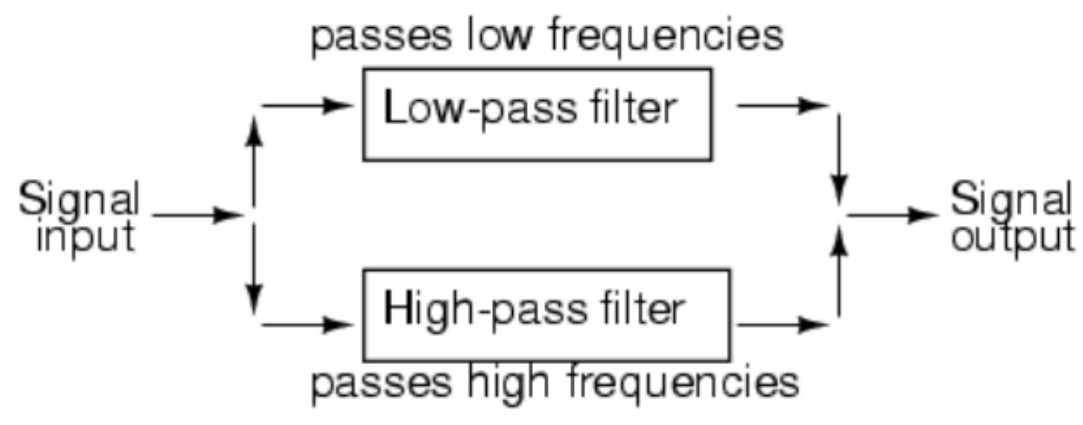

Figure 1.Concept of band stop circuit

The R-C band stop filter is similar to a band pass filter in which the shunt arm is replaced by the series arm and the series arm is replaced by the shunt one.

Flexibility and tunability are the big advantages of OTAs. The output current io of the ideal COTA can be expressed by equation (1)

$$
\text { io }=\operatorname{gm}(\mathrm{vp}-\mathrm{vn})
$$

Where gm is the transconductance, $\mathrm{vp}$ and $\mathrm{vn}$ are positive and negative input terminals respectively. The ideal OTA has infinite output resistance. All of io flows in the external capacitive load and none flows in the OTA's own output resistance .Towards increasing the OTA output resistance, the current mirrors are cascoded. Cascode amplifier configuration improves gain due to Band output resistance and bandwidth due to reduced Miller capacitance.

\section{PROPOSED COTA BASED BAND STOP FILTER}

The Cascode Operational transconductance amplifier (COTA) circuit is used to design BSF. The proposed BSF is simulated using $0.9 \mathrm{~V}$ at $32 \mathrm{~nm}$.

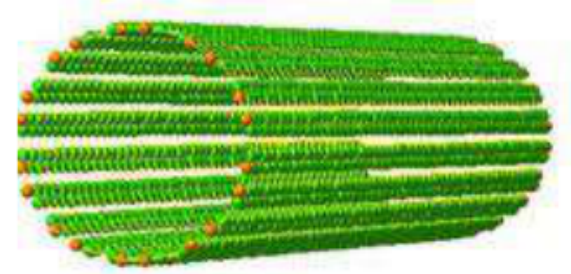

Single wall

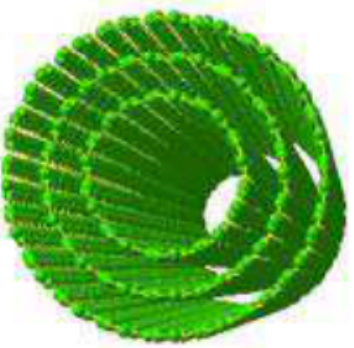

Multiwall

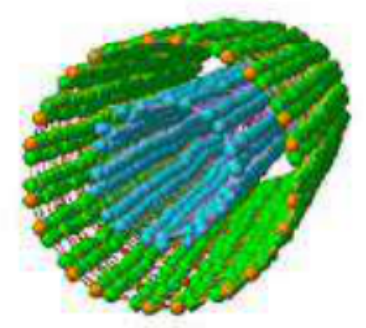

Double wall 

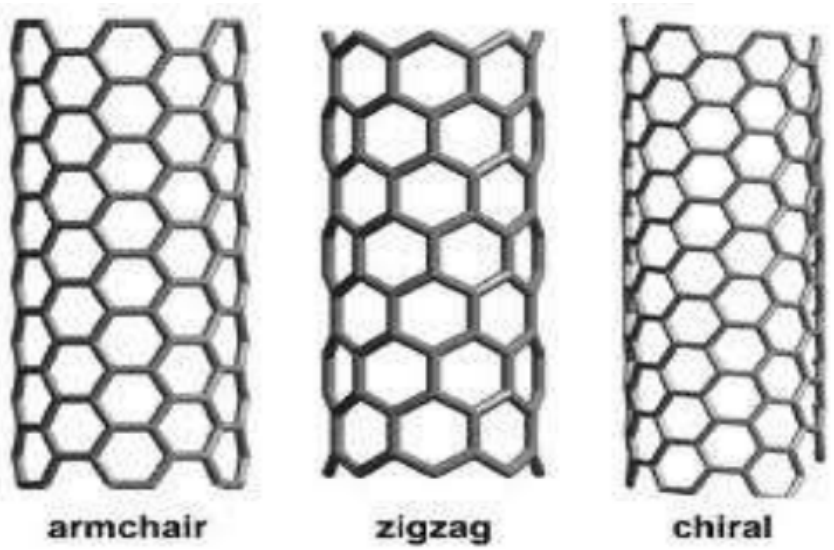

Figure 2. (a) Different types (b) different configurations of SWCNT

The carbon nanotubes exist in two forms: (i) Single wall carbon nanotube (SWCNT) and (ii) multiwall carbon nanotube as shown in Figure 1(a). SWCNT are actually tubes of graphite that are normally capped at the ends. They can be visualized as a layer of graphite rolled into a seamless cylinder [8]. Their diameter is around $1 \mathrm{~nm}$ and length a few microns. They are superior to MWCNT, however, are costlier. MWCNT appear like a coaxial assembly of SWCNTs, like a coaxial cable. They diameter of MWCNT ranges from 5-50 nm and the inter layer spacing is $3.4 \AA$. They are easy to produce in large quantity. However, the structure is complex and the structural imperfections may diminish their unique properties [10]. The wrapping of graphite sheets in SWCNT can be represented by a pair of indices $(n, m)$, called as the chirality vector or roll-up vector, as shown in Figure 1(b). There are three types of SWCNTs based on the chiral vector and chiral angle $(\theta)$.

Figure 3. Shows the schematics of one of the important application of CNT, that is, carbon nanotube field effect transistor (CNTFET). In a CNTFET, the channel is made up of parallel combination of SWCNTs. The source and drain regions are highly doped regions and the CNT channel is undoped. The important advantages of CNTFET include 1D ballistic transport of charge carriers, high mobility, large drive current and very low power consumption [11].

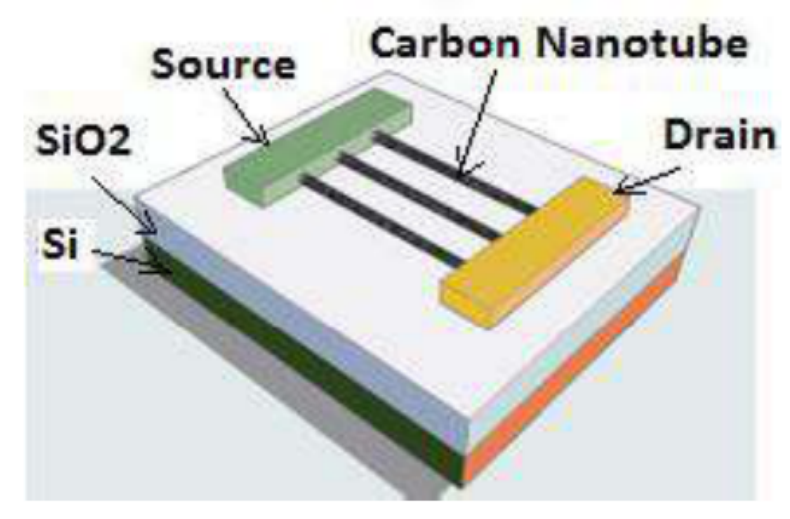

Figure 3. Schematic of a CNTFET 


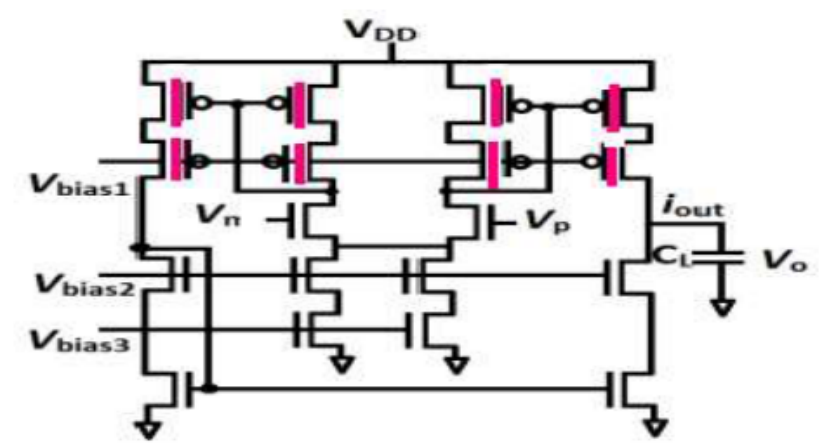

Figure 4. COTA [9]

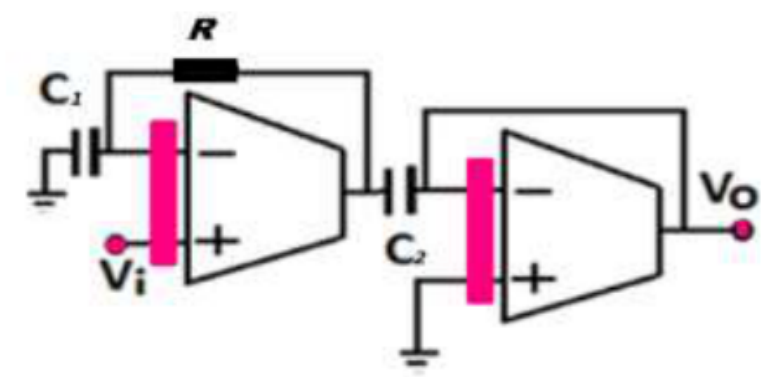

Figure 5. Proposed Band Stop Filter

The active filter design using operational amplifier has a serious limitation over the applications in the Band frequency regions. To overcome these limitations active filters using COTAs are popular due to the salient features of COTA such as, the adjustable transconductance (gm) over wide range of bias current, excellent matching between amplifiers, the linearity of transconductance with bias current, controlled impedance buffers and Band output signal to noise ratio, which popularizes COTA in active filter design. Filters can be readily built using COTAs. Considerable flexibility in controlling those specific filter characteristics that are usually of interest is possible with COTAs.

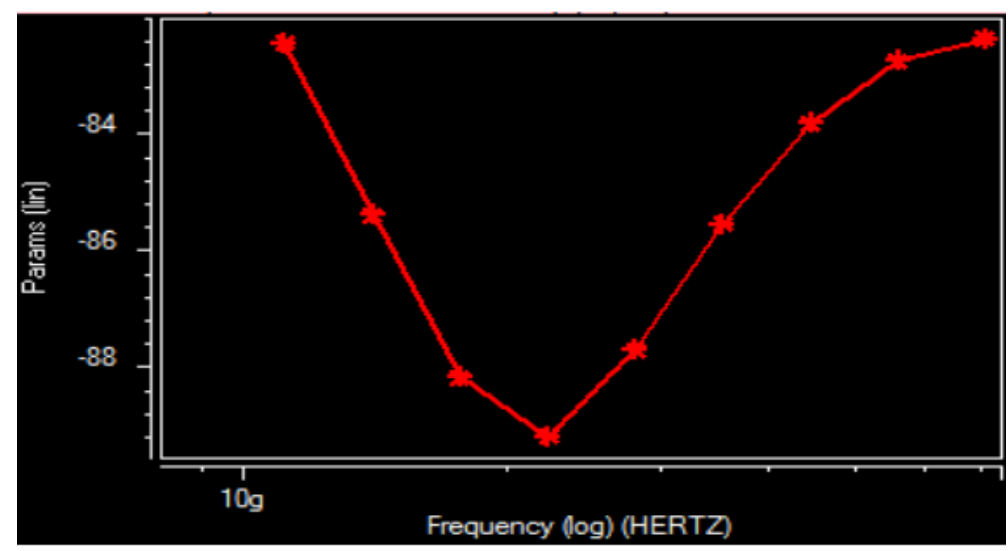

Figure 6. Frequency response of Proposed BSF 


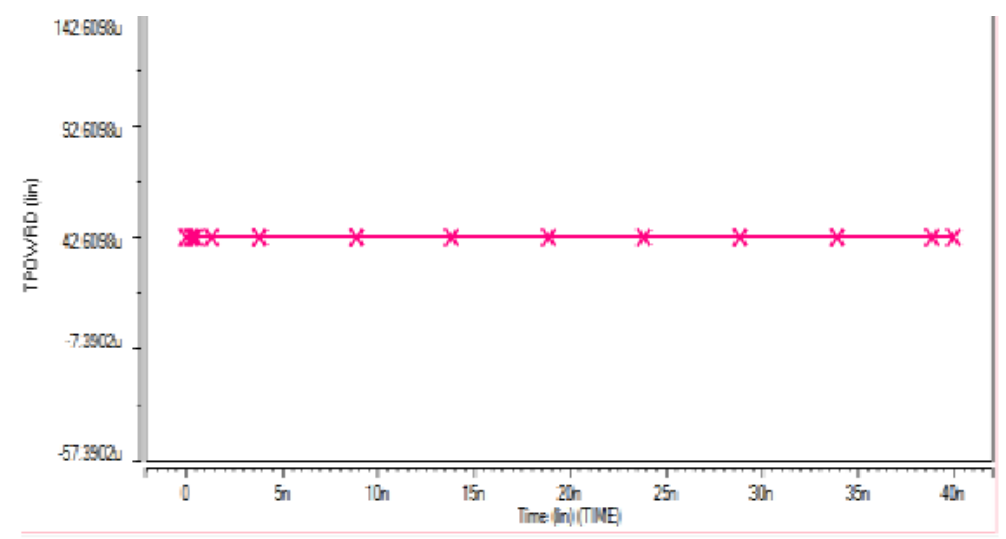

Figure 7. Average Power of Proposed BSF

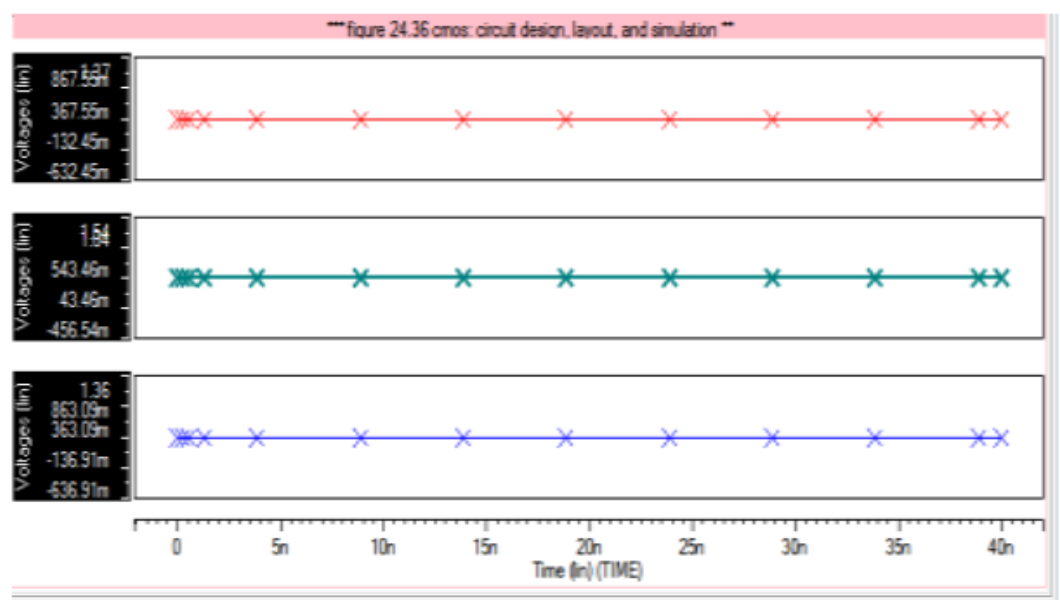

Figure 8. Biasing Currents used in Proposed BSF

\section{CONCLUSION}

Cascode Operational transconductance amplifiers (COTA) based Band stop filter is designed and simulated at $45 \mathrm{~nm}$ technology using $0.9 \mathrm{~V}$ for analog applications. It has been designed and simulated using novel carbon nanotube based MOS structures and conventional MOSFETs. The proposed structure is useful in Nano electronic circuits. It is observed that the proposed BPF is also consuming low power of $42 \mu \mathrm{W}$. It is shown from the frequency response characteristics of Band Stop filter that the filtering can be performed successfully over the designed range with a reasonable accuracy.

\section{REFERENCES}

[1] RCA Solid-state Division, Data Book, Linear Integrated circuits, File No.475, Mar.1975.

[2] Sridevi.V, Jayanthy.T., "Design of Active Filters using CNTFET Opamp", International Journal of Soft Computing and Engineering”, Volume -1, Issue -3, July 2011.

[3] Li Tianwang, Ye Bo and Jiang Jinguang," A novel fully differential telescopic Cascode Operational transconductance amplifier", Journal of Semiconductors, IOP Science, vol. 30, no. 8, August 2009.

[4] Xuguang Zhang and Ezz I. El-Masry,"A Novel CMOS COTA Based on Body-Driven MOSFETs and its Applications in COTA-C Filters", IEEE Transactions on Circuits and Systems-I: Regular Papers, vol. 54, no. 6, pp 1204-1212, June 2007.

[5] A.K. Kureshi, M. Hasan, "Comparison of performance of Carbon nanotube FET and bulk CMOS based 6T SRAM cell in deep submicron", Microelectronics Journal 40 (6), page 979-982, 2009.

[6] S. A. Loan, M. Nizamuddin et.al., "Band Performance Carbon Nanotube based Cascode Cascode Operational trans conductance amplifiers", World Congress on Engineering, 2014, Vol I, July 2 - 4, 
[7] M. Nizamuddin, S. A. Loan, et.al., "Design, simulation and the comparative analysis of CNTFET based multistage operational amplifiers", Journal of Nanoelectronics and Optoelectronics, 2017, Vol-12,

[8] Rajinder Tiwari, R. K. Singh, Department of Electronics \& Communication Engineering, Kumaon Engineering College, Dawarahat (Almora), Uttarakhand, Ganga Ram Mishra, Department of Electronics \& Communication Engineering, Amity School of Engineering \& Technology, Amity University Uttar Pradesh, Lucknow ,A New Approach for Design of Cmos Based Cascode Current Mirror for Asp Applications, International Journal of Electronics and Communication Engineering \& Technology (IJECET), 2011, Volume:2, Issue: 2, Pages: 1-7.

[9] R. K. Singh And Ashish Dixit, Data Transmission With Gbits Speed Using Cmos Based Integrated Circuits For Opto-Electronic Interfaces And Applications, International Journal of Computer Engineering \& Technology (IJCET), 2013, Volume:4,Issue:3,Pages:188-203.

[10] M. Nizamuddin, et.al., "Carbon Nanotube based three stage Operational Amplifiers, A simulation Study", Journal of Materials Today Proceedings, 2015, Volume 3, Issue 2, 2016, Pages 449-453,

[11] M. Nizamuddin, S. A. Loan, et.al., "Design, simulation and comparative analysis of CNT based Cascode Cascode Operational transconductance amplifiers", Nanotechnology, IOP Publishing Ltd ,October 2015, Volume 26, Number 39, 02

[12] S. A. Loan et al., Design of a novel Band gain carbon nanotube based Cascode Operational transconductance amplifier, Proc. IMECS (2014) pp. 797-800.

[13] S. A. Loan, M. Nizamuddin et.al., "Design and Comparative Analysis of Band Performance Carbon Nanotube-Based Cascode Operational transconductance amplifiers", NANO: World Scientific Publisher, 2015, Vol. 10, No. 3,

[14] Fahad Ali Usmani, Mohammad Hasan,"Carbon nanotube field effect transistors for high performance analog applications: An optimum design approach ",Microelectronics Journal, 41 (2010)

[15] J. Yeo, Compact CPW Slot Resonators and A Band International Journal of Civil Engineering and Technology (IJCIET 410-416.

[16] J.Deng, H.S.P.Wong, "A compact SPICE model for carbon nanotube field effect transistors including non-idealities and its application - Part II: Full device model and circuit performance benchmarking “, IEEE Transactions on Electron Devices 54 (12) (2007) 3195-3205.

[17] A.Javey et.al, "Self-Aligned ballistic molecular transistors and electrically parallel nanotube arrays", Nano Letters 4(2004)1319-22.

[18] J.Appenzeller, "Carbon nanotubes for high performance electronics (Invited paper)", Proceedings of the IEEE 96(2) (2008) 206.

[19] Sarah Khwaja," Design and Simulation of High performance High Pass filter at $45 \mathrm{~nm}$ technology node", International Journal of Innovative Research in Electrical, Electronics, Instrumentation and Control Engineering Vol. 7, Issue 7, July 2019.

[20] Sarah Khwaja," Simulation Study of High Performance Low Pass filter at Nanoscale", International Journal of Advanced Research in Computer and Communication Engineering Vol. 8, Issue 8, August 2019 .

[21] Sarah Khwaja,"Design and Performance Analysis of Low Power Band Pass Filter at $32 \mathrm{~nm}$ Technology", International Journal of Innovative Research in Electrical, Electronics, Instrumentation and Control Engineering Vol. 7, Issue 9, September 2019. 